\title{
Fenologia reprodutiva e produção de sementes em Araucaria angustifolia (Bert.) O. Kuntze ${ }^{1}$
}

\author{
ADELAR MANTOVANI ${ }^{2}$, L. PATRÍCIA C. MORELLATO ${ }^{2,4}$ e MAURÍCIO S. DOS REIS ${ }^{3}$
}

(recebido: 4 de julho de 2003; aceito: 16 de setembro de 2004)

\begin{abstract}
Reproductive phenology and seed production of Araucaria angustifolia (Bert.) O. Kuntze). The knowledge about the reproductive phenology of Araucaria angustifolia has applications to the seed harvest for "ex situ" conservation, for commercial use of the seeds and to understand the dynamic and regeneration of its natural populations. The reproductive phenology and seed production of A. angustifolia were evaluated for a natural population occurring at Parque Estadual Campos de Jordão, São Paulo State, Brazil. The present study investigates: (i) how is the reproductive cycle and the phenological pattern of araucaria? (ii) how is the potential of seed production and its variability? Sixty adult plants (30 males and 30 females) were marked for phenological observations. Seed production was estimated based on the number of female plants in a 10 ha area, number of cones per plant, number of seeds per cone and seed weight. The reproductive cycle of A. angustifolia extended for 20 to 24 months, from the observation of the first cone to seed fall. The pollination occurred between September and October and seed maturation and seed fall from March to June. Seed production was significantly different between years (117 kg.ha-1 in 2001 e $160 \mathrm{~kg} \cdot \mathrm{ha}^{-1}$ in 2002). The duration of ripe seed offer was also different between years. The variation observed in the amount and availability of seeds suggests that the observation of reproductive phenology and estimation of seed production are important to guide strategies for conservation and management of this resource.
\end{abstract}

Key words - Araucaria angustifolia, Araucaria forest, reproductive phenology, seed production

RESUMO - (Fenologia reprodutiva e produção de sementes em Araucaria angustifolia (Bert.) O. Kuntze). O estudo da fenologia reprodutiva de $A$. angustifolia tem aplicação na coleta de sementes destinadas à conservação de germoplasma, à obtenção de sementes para fins comerciais e ao entendimento da dinâmica de regeneração das populações naturais. Este trabalho teve como objetivo investigar a fenologia reprodutiva em uma população natural de A. angustifolia localizada no Parque Estadual Campos do Jordão, SP, procurando entender: (i) Qual é o seu ciclo reprodutivo e o comportamento fenológico? (ii) Como varia e qual o potencial de produção de sementes desta espécie? Para o acompanhamento da fenologia reprodutiva foram marcados 60 indivíduos (30 masculinos e 30 femininos), observados de novembro de1999 a agosto de 2002 . A produção de sementes foi estimada com base na contagem do número de plantas femininas, número de estróbilos por planta, número de sementes por estróbilo e peso de sementes. O ciclo reprodutivo da A. angustifolia foi de 20 a 24 meses, do aparecimento dos estróbilos até a queda das sementes. A polinização ocorreu entre setembro e outubro e a maturação e queda das sementes de março a junho. A produção de sementes mostrou diferença significativa entre os anos $\left(117 \mathrm{~kg} \cdot \mathrm{ha}^{-1} \mathrm{em} 2001 \mathrm{e} 160 \mathrm{~kg} . \mathrm{ha}^{-1} \mathrm{em}\right.$ 2002) e a duração da oferta foi distinta entre anos. As variações na quantidade e duração da oferta de sementes sugerem que observações do comportamento reprodutivo são indispensáveis para a conservação e manejo adequado deste recurso.

Palavras-chave - Araucaria angustifolia, fenologia reprodutiva, floresta de araucária, produção de sementes

\section{Introdução}

A Floresta Ombrófila Mista, também conhecida como Floresta de Araucária, faz parte do bioma da Mata Atlântica (Decreto Lei 750/1993), considerado como

1. Parte da Tese de Doutorado de A. Mantovani, Pós-graduação em Biologia Vegetal, Universidade Estadual Paulista, Instituto de Biociências, Departamento de Botânica.

2. Universidade Estadual Paulista, Instituto de Biociências, Departamento de Botânica, Grupo de Fenologia de Plantas e Dispersão de Sementes, Caixa Postal 199, 13506-900 Rio Claro, SP, Brasil.

3. Universidade Federal de Santa Catarina, Centro de Ciências Agrárias, Departamento de Fitotecnia, Caixa Postal 476, 88040-900 Florianópolis, SC, Brasil.

4. Autor para correspondência: pmorella@ rc.unesp.br detentor de altíssimos níveis de diversidade, apesar de intensamente explorado (Lima \& Capobianco 1997). No início do século XX cerca de $35 \%$ da cobertura vegetal dos estados do sul do Brasil estavam representados pela Floresta de Araucária. Atualmente, estima-se que os remanescentes ocupem entre $1 \%$ a $4 \%$ da área original (Lima \& Capobianco 1997, Guerra et al. 2002). Devido a esta drástica redução em sua área de ocorrência, a Floresta de Araucária é considerada em estado crítico em termos de conservação (Dinerstein et al. 1995, Biodiversity Support Program 1995).

A espécie Araucaria angustifolia (Bert.) O. Kuntze é o principal componente da Floresta de Araucária. Pertencente à família Araucariaceae é a única epécie de seu gênero com ocorrência natural no 
Brasil e está na categoria de vulnerável na lista de espécies ameaçadas da IUCN (Hilton-Taylor 2000). Essa espécie fornece principalmente madeira, mas também outros produtos como sementes para alimentação humana e da fauna nativa além de resina (Inoue et al. 1984, Carvalho 1994). A madeira da araucária representou um importante produto de exportação nos anos 60 (Reitz et al. 1978) e suas características silviculturais têm revelado grande potencial para reflorestamentos comerciais (Embrapa 1988, Guerra et al. 2002).

A área de ocorrência natural de A. angustifolia ocupava, no sul do Brasil, grande parte dos estados do Paraná, Santa Catarina e Rio Grande do Sul. Agrupamentos menores eram encontrados nos estados de São Paulo, Minas Gerais e Espírito Santo (Backes 1999). A área de ocorrência estava limitada a um quadrilátero formado pelos paralelos $19^{\circ} 15^{\prime}$ e $31^{\circ} 30^{\prime} \mathrm{S}$ e

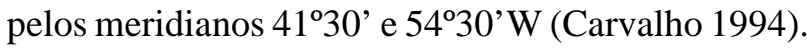

Apesar da importância econômica e ecológica da araucária, poucos trabalhos têm enfocado a biologia desta espécie em formações naturais (Solórzano Filho 2001). Na maioria dos casos, apenas informações gerais sobre fenologia da espécie são mencionadas e indicam a emissão de estruturas reprodutivas (estróbilos) de agosto a janeiro e a produção de sementes de março a setembro, no Paraná, de abril a julho em São Paulo e Santa Catarina, e de abril a agosto no Rio Grande do Sul (Carvalho 1994). Os trabalhos que abordaram a biologia reprodutiva apresentam algumas informações conflitantes com relação ao ciclo de produção de sementes. Burlingame (1914) mencionou que a vida de um cone feminino não é maior que dois anos; Shimoya (1962) relatou que o desenvolvimento a partir do carpelo primordial até a semente demora aproximadamente quatro anos e Mattos (1994) registrou que o tempo de formação de uma pinha desde a diferenciação celular é de mais de três anos.

O detalhamento da fenologia reprodutiva de A. angustifolia é de grande importância tanto econômica como ecológica, com aplicação direta na coleta de sementes destinadas à conservação de germoplasma, na obtenção de sementes para fins comerciais e no entendimento da dinâmica e regeneração de populações naturais.

Este trabalho tem como objetivo geral investigar a fenologia reprodutiva em uma população natural de A. angustifolia. As perguntas a serem respondidas são: (i) Qual é o ciclo reprodutivo e o comportamento fenológico de A. angustifolia? (ii) Como varia a produção de sementes entre as plantas? e (iii) Qual o potencial de produção de sementes para esta espécie no local de estudo?

\section{Material e métodos}

Área de estudo - Este trabalho foi realizado em uma floresta contínua com ocorrência natural de Araucaria angustifolia (Bert.) O. Kuntze (Araucariaceae) localizada no Parque Estadual de Campos do Jordão, do Instituto Florestal, Município de Campos do Jordão, Estado de São Paulo, na Serra da Mantiqueira (22 $45^{\prime} \mathrm{Se} 45^{\circ} 30^{\prime} \mathrm{W}$ ) e altitude de $1.450 \mathrm{~m}$. Abrangendo cerca de 8.341,86 ha, esta área foi explorada há mais de 45 anos, quando foi transformada em Parque Estadual. A área apresenta topografia acidentada com agrupamento denso de araucária limitado na borda superior por campo.

Segundo a classificação de Koeppen, o clima da região é do tipo $\mathrm{Cfb}$, ou seja, clima subtropical de altitude, mesotérmico e úmido, sem estiagem. Na região de Campos do Jordão a precipitação anual é de $2.000 \mathrm{~mm}$, com um período menos úmido de abril a setembro e um período chuvoso de outubro a março, quando ocorre $73 \%$ de toda a precipitação anual (figura 1). O mês mais frio é julho, com temperatura média de $8,9^{\circ} \mathrm{C}$ e mínima absoluta de $7,2^{\circ} \mathrm{C}$ negativos. O mês mais quente é predominantemente fevereiro, cuja temperatura média é de $17,7^{\circ} \mathrm{C}$, com máxima absoluta de $27,2^{\circ} \mathrm{C}$ (Seibert et al. 1975).

Para este estudo foi demarcada uma área de 10 ha de floresta onde foram contados, mapeados e sexados todos os indivíduos reprodutivos de Araucaria angustifolia (Araucariaceae), mediante pontos georeferênciados com GPS-Garmin48 (Global Position System). Sendo a araucária uma espécie dióica, uma das dificuldades nas observações de campo é a definição do sexo das plantas. A presença das estruturas reprodutivas foi a única forma de identificação do sexo no campo. Para plantas femininas esta definição foi facilitada pelo fato de sempre apresentarem estróbilos em diferentes fases, independente do período do ano. Para plantas masculinas a definição foi facilitada durante a fase de liberação de pólen e logo depois, pois as estruturas permanecem secas na planta por pelo menos dois meses.

Fenologia - Dos indivíduos reprodutivos presentes na área, 60 (30 masculinos e 30 femininos) foram escolhidos ao acaso e acompanhados quanto à fenologia reprodutiva, de novembro de 1999 a agosto de 2002. A presença ou ausência de estróbilos foi anotada a partir da visualização com binóculo. Para os anos de 2001 e 2002 foi avaliada a intensidade de emissão de estróbilos masculinos, atribuindo notas de zero a quatro, de acordo com método de avaliação fenológica proposto por Fournier (1974). As observações foram mensais no período de liberação de pólen e bimestrais no restante do ciclo. Foram realizadas coletas de três estróbilos femininos, a cada dois meses, para acompanhar o seu desenvolvimento, através de medidas do diâmetro no sentido transversal ao eixo de inserção dos estróbilos. Esses estróbilos foram 
coletados de nove plantas dentro da área de estudo. Foram tomadas as medidas de altura, diâmetro a altura do peito e calculada a área basal dos 60 indivíduos da avaliação fenológica.

Produção de sementes - No ano de 2001, a produção de estróbilos por planta foi avaliada com base na contagem de estróbilos de 40 indivíduos. Para a contagem do número de

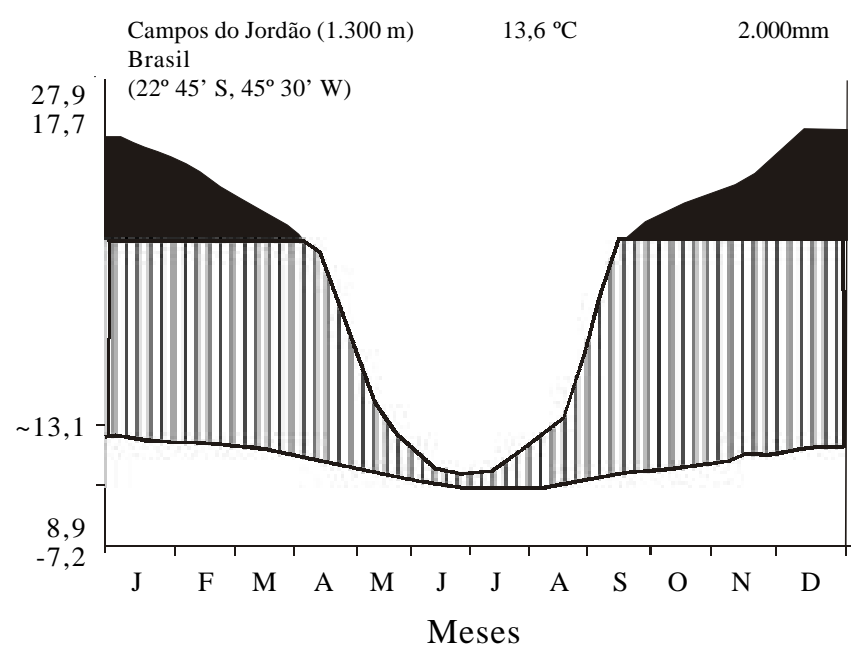

Figura 1. Diagrama climático para o Parque Estadual Campos do Jordão, SP, construído segundo Walter \& Lieth (19601967 apud Longman \& Jeník 1974), correspondente a um período de 30 anos (1969-1999). Curva inferior: temperatura média mensal (intervalos $10^{\circ}$ no eixo y); curva superior: precipitação média mensal total (intervalos $20 \mathrm{~mm}$ no eixo y, exceto área negra, onde a escala é reduzida a 1/10). Área com traços: períodos úmidos; área negra: períodos super-úmidos, precipitação superior a $100 \mathrm{~mm}$ por mês). Acima, no gráfico: local e altitude, temperatura média anual e precipitação média anual (mm). Temperaturas à esquerda, lidas a partir do topo: máxima absoluta, média das máximas do mês mais quente, variação média diária, média das mínimas do mês mais frio e mínima absoluta. Fonte: Estação meteorológica do Parque Estadual Campos do Jordão.

Figure 1. Climatic diagram for the Parque Estadual Campos do Jordão, SP, according to Walter \& Lieth (1960-1967 apud Longman \& Jeník 1974), for a 30 years period (1969-1999). The lower curve shows the mean monthly temperature $\left(10^{\circ} \mathrm{C}\right.$ intervals on the y axis); the upper curve monthly rainfall totals (20 mm intervals on the axis y, except in the black zone when the scale is reduced by $1 / 10$ ). Shaded zone: humid period; black zone wet period, when the precipitation is over $100 \mathrm{~mm}$ per month. Shown above are the local altitude, mean annual temperature and annual rainfall $(\mathrm{mm})$. Temperatures on the left, reading from the top, are the absolute maximum, the mean daily maximum for the warmest month, the mean diurnal range, the mean daily minimum for the coolest month, and the absolute minimum. Source: Meteorological Station at Parque Estadual Campos do Jordão. sementes por estróbilo, foram coletados 31 estróbilos maduros de 16 plantas. Uma amostra aleatória de 560 sementes desses estróbilos foi utilizada para estimativa da massa. Em 2002, para a estimativa de produção de estróbilos por planta, foram contados os estróbilos de 30 plantas. De cada uma dessas plantas foram coletados três estróbilos maduros, dos quais foram tomadas medidas de diâmetro, massa, número de sementes, de escamas não fertilizadas (pinhões chochos) e de escamas estéreis (falhas). Uma amostra de 20 sementes maduras de cada estróbilo foi utilizada para pesagem individual. Outra amostra de dez sementes de seis plantas foi coletada para pesagem dos componentes da semente, a saber: testa, endosperma e embrião. A predação pré-dispersão foi avaliada em 6.332 sementes de 81 estróbilos maduros retirados de 26 plantas das 30 inicias. Foram consideradas predadas as sementes que apresentavam orifícios deixados pela larva de Laspereysia araucarie (Lepidoptera), principal predador de sementes de araucária (Gallo et al. 1988).

A produção de sementes foi estimada da seguinte forma: Produção de sementes em $\mathrm{kg} / \mathrm{ha}=$ [número de plantas femininas/ha $\times$ número médio de estróbilos por planta $\times$ número médio de sementes por estróbilo $\times$ peso médio das sementes $\left.(\mathrm{g}) \cdot 1000^{-1}\right]$.

Análise estatística - As análises estatísticas geralmente seguiram Zar (1996), exceto quando indicado. A produção de sementes por indivíduo foi submetida à análise de variância (Anova). Para a produção de 2001 foram testadas as diferenças de produção entre 14 plantas das 16 iniciais e, para 2002, os dados de produção de sementes foram submetidos à Anova hierarquizada, testando diferenças de massa de sementes dentro do estróbilo, de estróbilos dentro de plantas e entre plantas (Steel et al. 1997), em 25 plantas das 30 iniciais. A prova de Wilcoxon foi utilizada para comparar as intensidades de emissão de estruturas reprodutivas masculinas entre anos, e a correlação de Kendall foi utilizada para relacionar as intensidades de emissão de cada indivíduo entre anos (Siegel 1975). Para o ano de 2002 foram feitas análises de correlação de Spearman $\left(r_{s}\right)$ entre as variáveis que caracterizam os indivíduos (altura e área basal) e aquelas que caracterizam a produção de sementes (número de estróbilos por indivíduo e número médio de sementes por estróbilos) para 28 plantas das 30 iniciais. As análises estatísticas foram realizadas com o auxílio do Software Statistica $A X$ versão 5.5 (2000).

\section{Resultados}

Foram amostrados 321 indivíduos de Araucaria angustifolia nos 10 ha demarcados (32 indivíduos.ha ${ }^{-1}$ ), dos quais, 146 eram femininos e 175 masculinos. Todas as plantas acompanhadas emitiram estróbilos durante o período de estudo. As observações fenológicas mostraram que o ciclo reprodutivo da araucária foi de 20 a 24 meses, sendo o início do ciclo caracterizado 
pelo aparecimento dos estróbilos e o final, pela produção de sementes (tabela 1). O ciclo considerado a partir da liberação de pólen foi de 19 meses.

Os estróbilos masculinos foram percebidos a partir do mês de fevereiro e, entre os meses de agosto e setembro, ocorreu a liberação do pólen (tabela 1). Os indivíduos masculinos produziram estróbilos nos dois anos de observação, com duração de sete meses do aparecimento à liberação do pólen. Os estróbilos masculinos são emitidos em ramos jovens (figura 2A), os quais normalmente não emitirão novos estróbilos no próximo ano. A intensidade de emissão de estróbilos masculinos na população foi semelhante entre os anos $(\mathrm{Z}=0,849 ; p=0,39 ; \mathrm{n}=30)$ e variou entre plantas, onde, em 2001 a distribuição das plantas em função da intensidade de emissão de estróbilos foi: oito, onze, oito e três plantas com intensidades 1, 2, 3 e 4, respectivamente. Em 2002 foi: nove, sete, oito e seis plantas com intensidades $1,2,3$ e 4 , respectivamente. A emissão de estróbilos masculinos variou entre plantas, mas apresentou tendência de repetição da intensidade para os mesmos indivíduos entre anos $(Z=2,74$; $p=0,006 ; \mathrm{n}=30$ ). Dos 30 indivíduos observados, $40 \%$ repetiram a mesma intensidade de emissão nos dois anos de observação.

Os estróbilos femininos, no início de seu desenvolvimento, ocupavam as extremidades dos ramos primários conectadas por um pequeno pedúnculo (figura 2B). Durante as observações os estróbilos estavam visíveis a partir dos meses de maio e julho (tabela 1). No entanto, é provável que o início de seu desenvolvimento tenha ocorrido junto com o dos estróbilos masculinos, em fevereiro. Durante os meses
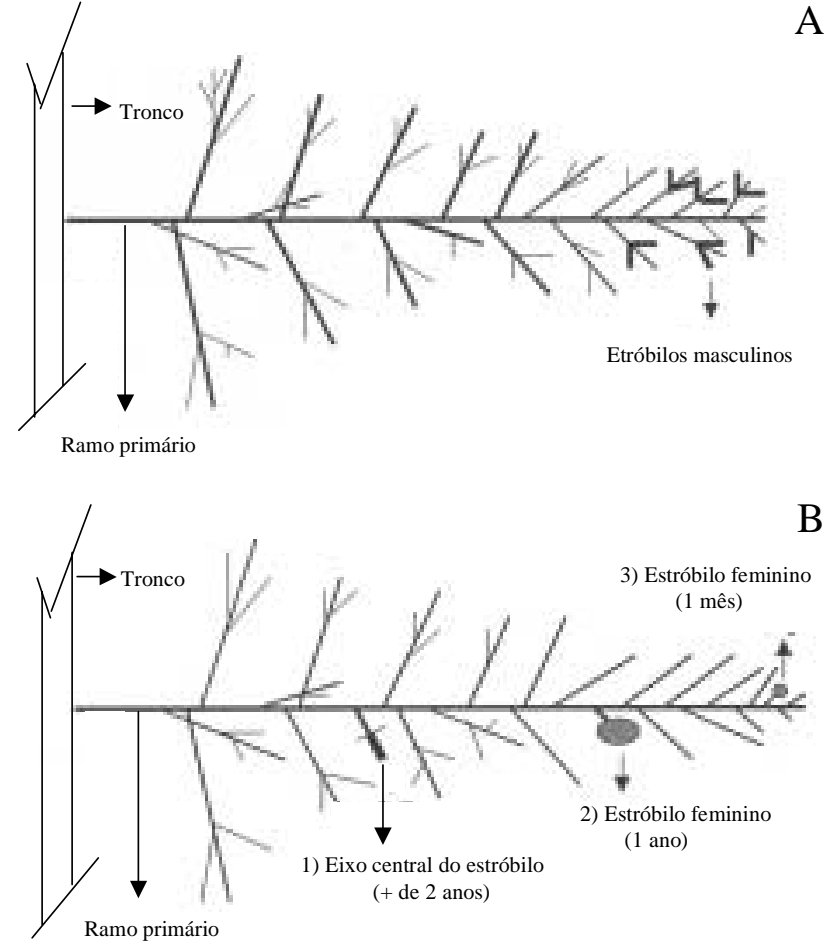

Figura 2. Organização de um ramo primário de Araucaria angustifolia. A - Posição dos estróbilos masculinos nos ramos mais jovens. $\mathrm{B}$ - ramo de indivíduo feminino indicando: 1 - presença do eixo central do estróbilo com mais de dois anos, depois da queda de sementes; 2 - estróbilo com um ano de idade; 3 - estróbilo com um mês de idade.

Figure 2. Arrangement of a primary branch of Araucaria angustifolia. A - male branch showing the cone location on the young branches. B - female branch: 1 - showing the presence of the cone axis after the seed fall; 2 - one-year cone; 3 - one-month cone.

A

Tabela 1. Ciclo reprodutivo de Araucaria angustifolia, no Parque Estadual Campos do Jordão, SP. A linha cheia refere-se ao ciclo reprodutivo iniciado no ano 2000 e a linha pontilhada ao ciclo reprodutivo iniciado no ano de 2001.

Table 1. Reproductive cycle of Araucaria angustifolia at Parque Estadual Campos do Jordão, SP. The bold line refers to the reproductive cycle initiated in 2001, and the dotted line refers to the reproductive cycle initiated in 2002.

2000

2001

2002

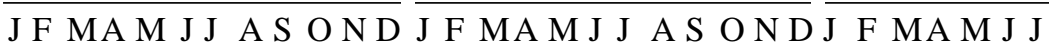

Estróbilos visíveis

- Crescimento

Liberação de pólen

Estróbilos visíveis

Estróbilo receptivo (polinização)

* Crecimento lento

Crescimento rápido

Maturação das sementes e queda 
de agosto e setembro ocorreu a polinização, período em que as escamas estavam abertas, propiciando a entrada de pólen. Depois da polinização, o crescimento do estróbilo produziu uma estrutura compacta que protege o pólen e o desenvolvimento do óvulo. A partir desta fase os estróbilos apresentaram crescimento lento durante 10 a 12 meses. Após esta fase, os estróbilos cresceram rapidamente até atingirem a maturação em torno do $24^{\circ}$ mês do ciclo (tabela 1). Os estróbilos femininos de diferentes estações puderam ser identificados pelo seu tamanho e pela distância entre eles, estando separados por sete a nove ramos vegetativos (figura 2B). O comportamento de crescimento dos estróbilos femininos pode ser representado com uma função logística (figura 3 ), onde podemos notar uma fase inicial de crescimento lento, seguida de uma fase de crescimento rápido até a maturação.

Produção de sementes - O período em que as sementes de araucária amadureceram iniciou em março e foi até início de junho em 2000, de março a maio em 2001 e de março a julho em 2002. A produtividade de sementes foi estimada em $117 \mathrm{~kg} \cdot \mathrm{ha}^{-1}$ e $160 \mathrm{~kg} \cdot \mathrm{ha}^{-1}$ para os anos de 2001 e 2002, respectivamente. Foram encontradas diferenças significativas entre os anos no número de estróbilos por planta, no número de sementes por estróbilo e no peso das sementes (tabela 2). A média do número de sementes por estróbilo foi maior em 2001 $(93,9)$ do que em 2002 (80,7). Entretanto, o número de estróbilos por planta e a massa de sementes apresentaram valores superiores para o ano de $2002 \mathrm{e}$, conseqüentemente, maior produção de sementes neste ano (tabela 2).

Para o ano de 2001, foram verificadas diferenças significativas com relação à massa de sementes entre indivíduos $(\mathrm{F}=118,27 ; \mathrm{Gl}=13 ; p<0,01)$. $\mathrm{O}$ desvio padrão associado às médias de massa de sementes foi semelhante entre indivíduos, sugerindo efeito da planta mãe para característica de massa de sementes (figura 4A). Para o ano de 2002, o desenho de coleta de sementes permitiu analisar a massa de sementes entre indivíduos, dentro de indivíduos e dentro de estróbilos. A análise de variância mostrou que houve variação significativa para o efeito da planta e de estróbilos com relação à massa das sementes (tabela 3). $\mathrm{O}$ efeito das sementes dentro dos estróbilos não foi significativo, indicando homogeneidade para esta característica. A maior parte da variação em peso de sementes de araucária foi associada à planta mãe (figura 4B).

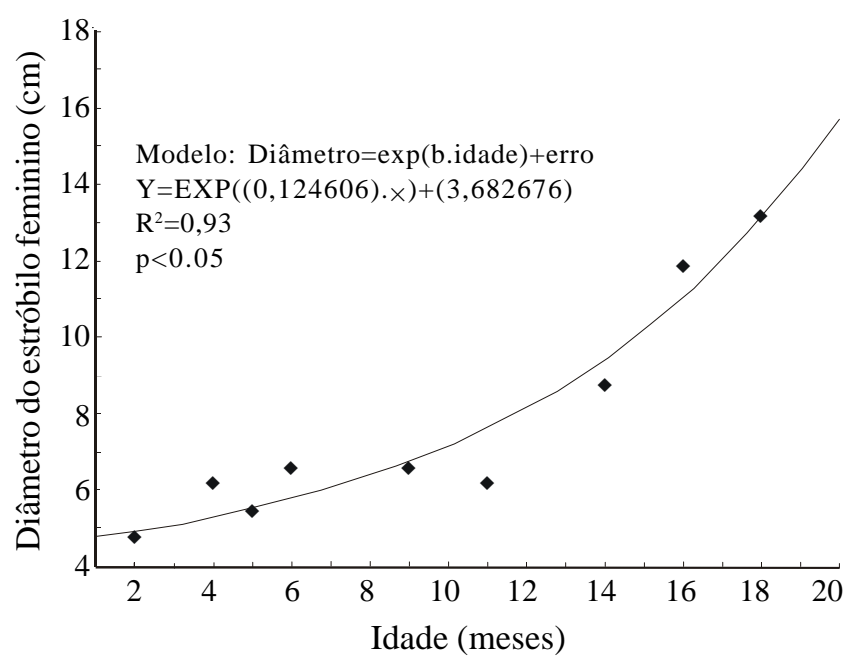

Figura 3. Modelo de crescimento em diâmetro de estróbilos femininos em função da idade para indivíduos de Araucaria angustifolia no Parque Estadual Campos do Jordão, SP.

Figure 3. Model for the diameter growth of female cones of Araucaria angustifolia in relation to time, Parque Estadual Campos do Jordão, SP.

Tabela 2. Valores médios e desvio padrão das variáveis utilizadas para estimativa da produção de sementes nos anos de 2001 e 2002 em uma população de Araucaria angustifolia localizada no Parque Estadual Campos do Jordão, SP.

Table 2. Mean values and standard deviation of the variables used to estimate seed production of Araucaria angustifolia in 2001 and 2002, Parque Estadual Campos do Jordão, SP.

\begin{tabular}{lccc}
\hline Ano & $\begin{array}{c}\text { Estróbilos } \\
\text { (número por planta) }\end{array}$ & $\begin{array}{c}\text { Sementes } \\
\text { (número por estróbilo) }\end{array}$ & $\begin{array}{c}\text { Massa das sementes } \\
(\mathrm{g})\end{array}$ \\
\hline 2001 & $13( \pm 7,6) \mathrm{n}=40$ & $93,9( \pm 21,0) \mathrm{n}=31$ & $6,58( \pm 1,4) \mathrm{n}=560$ \\
2002 & $19,5( \pm 9,4) \mathrm{n}=30$ & $80,7( \pm 25,2) \mathrm{n}=90$ & $7,0( \pm 1,6) \mathrm{n}=1800$ \\
t-teste & $\mathrm{t}=-3,49$ & $\mathrm{t}=2,69$ & $\mathrm{t}=-4,48$ \\
entre anos & $\mathrm{gl}=67$ & $\mathrm{gl}=123$ & $\mathrm{gl}=2868$ \\
& $\mathrm{p}<0,001$ & $\mathrm{p}=0,008$ & $\mathrm{p}<0,001$ \\
\hline
\end{tabular}



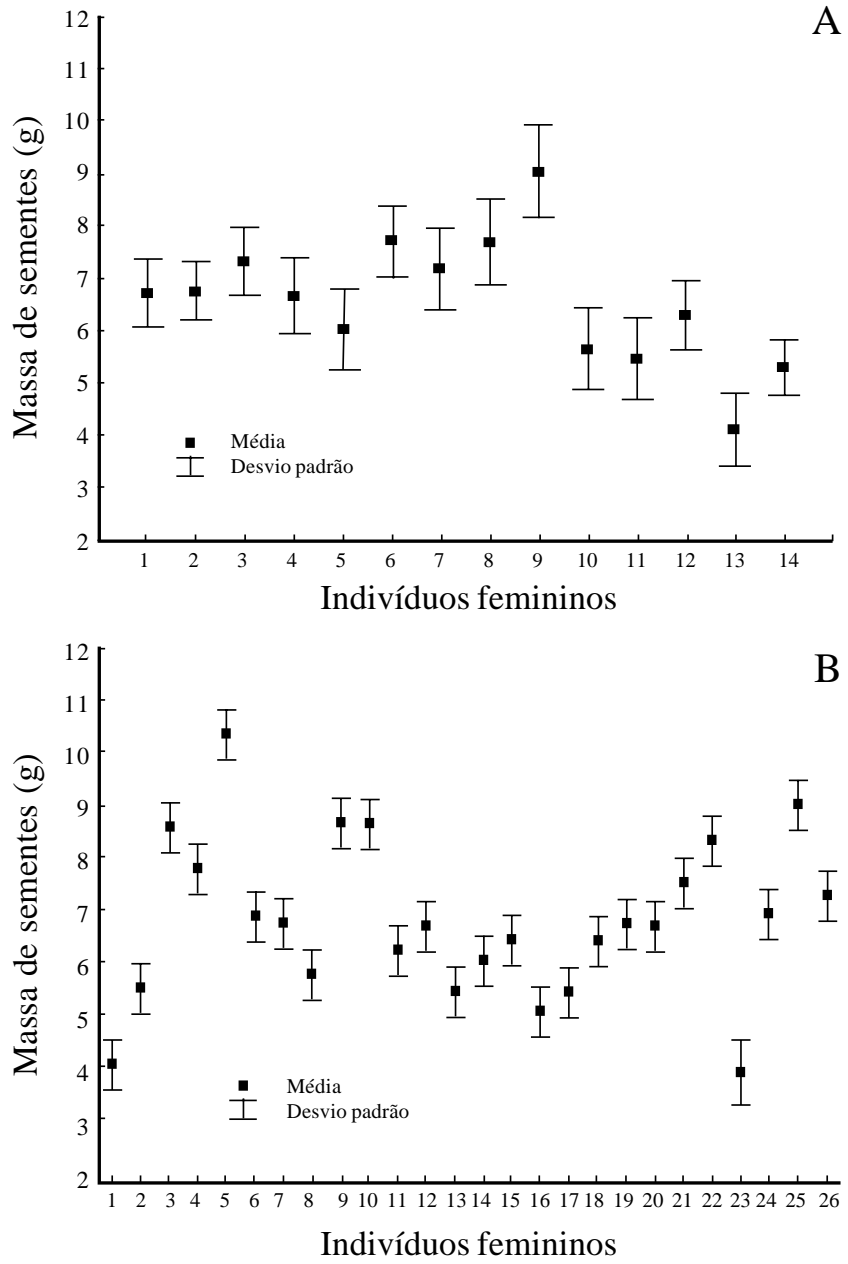

Figura 4. Média e desvio padrão da massa de sementes de Araucaria angustifolia produzidas por diferentes indivíduos (A) em 2001 e (B) em 2002, Parque Estadual de Campos do Jordão.

Figure 4. Mean mass of the Araucaria angustifolia seeds produced by different individuals (A) during 2001 and (B, C) produced during 2002, Parque Estadual Campos do Jordão, SP.

Foram encontradas correlações significativas entre número de estróbilos femininos por planta e a área basal $\left(\mathrm{r}_{\mathrm{s}}=0,48 ; p=0,008 ; \mathrm{n}=28\right)$ e a altura $\left(\mathrm{r}_{\mathrm{s}}=0,58\right.$; $p=0,001 ; \mathrm{n}=28)$, indicando maior capacidade de produção em plantas mais desenvolvidas. No entanto, não houve correlação significativa entre a média de peso das sementes de cada planta com a área basal $\left(\mathrm{r}_{\mathrm{s}}=0,03\right.$; $p=0,86 ; \mathrm{n}=24)$ e a altura $\left(\mathrm{r}_{\mathrm{s}}=0,18 ; p=0,46 ; \mathrm{n}=19\right)$ das plantas.

Os estróbilos femininos são compostos por sementes, escamas não fertilizadas (pinhões chochos), escamas estéreis e um eixo central. Foram encontradas, em média, 605 elementos (férteis + estéreis)
Tabela 3. Resumo da análise de variância hierarquizada para massa de sementes produzidas no ano de 2002, por indivíduos reprodutivos de Araucaria angustifolia no Parque Estadual Campos do Jordão, SP.

Table 3. Resume of nested analysis of variance for seed mass of Araucaria angustifolia, for seeds produced in 2002, Parque Estadual Campos do Jordão, SP.

\begin{tabular}{lccccc}
\hline $\begin{array}{l}\text { Causas de } \\
\text { variação }\end{array}$ & Gl & SQ & QM & F & P \\
\hline Plantas & 24 & 2877,641 & 119,9027 & 3,366 & $<0,001$ \\
$\begin{array}{c}\text { Estróbilos } \\
\quad(\text { plantas) }\end{array}$ & 48 & 1709,671 & 35,618 & 71,8252 & $<0,001$ \\
$\begin{array}{c}\text { Sementes } \\
\quad(\text { estróbilos) }\end{array}$ & 87 & 41,414 & 0,47602 & 0,9281 & 0,666 \\
\begin{tabular}{c} 
Erro \\
\hline
\end{tabular} & 2088 & 1070,871 & 0,5129 & & \\
\hline
\end{tabular}

constituintes da estrutura reprodutiva feminina, dos quais $13,4 \%$ sementes, $1,6 \%$ escamas férteis não fertilizadas ou abortadas (pinhões chochos) e $85 \%$ de escamas estéreis. Nesta população, a cada seis elementos estéreis ocorreu um fértil. Em média, 41,8\% do peso fresco dos estróbilos foram compostos por sementes, $50,7 \%$ por escamas estéreis e não fertilizadas e $7,5 \%$ pelo eixo central do estróbilo. A população estudada apresentou razão semente/escama estéril de $0,16(\mathrm{n}=94$; $\mathrm{DP}=0,050)$ e razão pinhões chochos/sementes de 0,08 $(\mathrm{n}=87 ; \mathrm{DP}=0,023)$.

As sementes de araucária são compostas pela testa, endosperma primário e embrião. O endosperma representou a maior porção em termos de peso fresco (71,2\%), seguido da testa (27\%) e do embrião $(1,8 \%)$. A correlação entre endosperma e testa foi significativa $(\mathrm{r}=0,80 ; \mathrm{p}<0,01 ; \mathrm{n}=119)$, bem como entre endosperma e embrião $(\mathrm{r}=0,65 ; \mathrm{p}<0,01 ; \mathrm{n}=119)$ e testa e embrião $(\mathrm{r}=0,51 ; \mathrm{p}<0,01 ; \mathrm{n}=119)$.

Predação pré-dispersão - Das 26 plantas avaliadas 11 apresentaram sementes predadas e com variação de $0,3 \%$ a $3,3 \%$ de predação. A predação de sementes pré-dispersão, causada pela lagarta Laspeyresia araucariae, foi de $0,7 \%$ de um total de 6332 sementes avaliadas.

\section{Discussão}

As observações fenológicas feitas neste estudo, durante dois anos e nove meses, mostraram que o ciclo reprodutivo em A. angustifolia foi de 19 a 20 meses da liberação de pólen até a maturação das sementes. A 
presença de apenas dois estágios de desenvolvimento de estróbilos femininos nas plantas indicou que o ciclo não passa de dois anos. Burlighame (1914) menciona que a duração do estróbilo feminino de A. angustifolia não é maior que dois anos, concordando com as observações deste estudo. Shimoya (1962) relata o ciclo reprodutivo em $A$. angustifolia de aproximadamente 30 a 32 meses a partir da polinização. Entretanto, o trabalho de Shimoya (1962) foi realizado com base em observações de plantas de jardim em Viçosa-MG, que se encontra fora da área de ocorrência natural desta espécie. Portanto, diferenças no ambiente devem ter influência no ciclo reprodutivo da araucária. $\mathrm{O}$ ciclo reprodutivo completo, observado para A. angustifolia neste estudo, foi similar ao de outros membros da família Araucariaceae como Agathis australis Steud., com ciclo de 16 meses (Owens et al. 1995) e para Wollemia nobilis W.G. Jones et al., com ciclo de 16 a 19 meses (Offord et al. 1999). Já para a família Pinaceae, o ciclo reprodutivo de espécies tropicais e temperadas varia de 2 a 3 anos, condicionado principalmente por fatores ambientais (Biswas \& Johri 1997).

A iniciação dos estróbilos masculinos e femininos de araucária ocorreu durante o verão. Comportamento semelhante foi descrito para outras coníferas como Pinus banksiana Lamb. (Despland \& Houle 1997). Estes autores sugerem que temperatura alta no início da fase reprodutiva favorece o desenvolvimento dos estróbilos. A posição erétil dos cones femininos de araucária nas extremidades dos ramos permite que a polinização seja mais eficiente, pois o grão de pólen pode cair com mais facilidade entre as escamas e mais próxima da micrópila. Esta característica também foi observada e descrita por Haines et al. (1984) para outras espécies da família Araucariaceae. A fertilização em A. angustifolia ocorre aproximadamente um ano depois da polinização (Burlinghame 1914, Shimoya 1962) e coincide com o início do rápido crescimento dos cones femininos, verificado neste estudo.

O período observado de produção de estróbilos masculinos foi de sete meses, não ocorrendo sobreposição na produção de estróbilos entre anos, como observado para as plantas femininas. A intensidade de produção de estróbilos masculinos foi semelhante entre os anos, mas variou entre plantas e tendeu a se manter constante dentro de uma mesma planta. Este comportamento sugere contribuição diferenciada entre plantas quanto à oferta de pólen.

A variação verificada entre anos no número médio de estróbilos femininos produzidos por planta pode refletir uma alternância entre anos de alta e baixa produção de sementes. Diferenças na quantidade de estróbilos femininos entre anos também são relatadas para Pinus resinosa Soland. (Mosseler et al. 1992) e para Juniperus communis L. (Ortiz et al. 2002) e é uma característica comum em outras coníferas (Powell 1977, Jordano 1991, Arista et al. 1997, Ortiz et al. 2002). Produção de sementes - Na área de estudo houve variação na duração de oferta de sementes. Enquanto nos anos de 2000 e 2002 a duração foi de praticamente cinco meses, para 2001 foi de apenas três meses. Esta variação na oferta de sementes tem implicações ecológicas, visto que este recurso é muito importante para a fauna, como destacado por Solórzano Filho (2001). Além da variação na duração de oferta, também foi verificada uma diferença na produção de sementes entre 2001 e 2002 da ordem de 30\%. Segundo Mattos (1994), a produção de sementes em $A$. angustifolia apresenta 2 a 3 anos de alta produção seguidos de 2 a 3 anos de baixa produção. Este estudo avaliou apenas dois anos de produção de sementes de araucária e, em virtude disto, é possível que a maior variação na produção não tenha sido amostrada. No entanto, as diferenças observadas indicam variação acentuada na oferta de sementes. Variação na produção de sementes entre anos é comum em outras gimnospermas, como relatado para Pinus banksiana (Houle \& Filion 1993). Estes autores relacionam essas variações às condições ambientais nas diferentes fases de desenvolvimento dos estróbilos. Este comportamento também tem sido associado à limitação de recursos na planta (Silvertown 1980, Kelly 1994).

As diferenças detectadas na massa das sementes entre plantas sugerem efeito materno para esta característica, já que o endosperma primário, em gimnospermas tem origem materna e na população estudada respondeu pela maior proporção da semente $(71,2 \%)$. Além do efeito da planta, foi observado efeito significativo associado ao estróbilo para a massa de sementes. Por outro lado, o peso das sementes dentro dos estróbilos não diferiu, refletindo uma distribuição homogênea de recursos dentro do estróbilo.

O período de oferta de sementes coincidiu com o período das menores precipitações e de temperaturas mais baixas. Esta característica releva a importância desta espécie para a fauna, já que este é um período de menor abundância de recursos alimentares na floresta, como relatado por Solórzano Filho (2001). Em outras espécies, em geral a dispersão de propágulos ocorre em um período do ano em que as condições ambientais são as melhores para o estabelecimento das novas plântulas (Janzen 1967, Primack 1987, Van Schaik et al. 1993). Levando em consideração que a araucária 
apresenta sementes recalcitrantes (Espindola et al. 1994), de baixa longevidade (Ferreira 1977), ou seja, não apresentam dormência, o período de oferta de sementes durante a estação mais fria e seca não seria o mais adequado para sua germinação e estabelecimento. No entanto, na área de estudo, o período das menores precipitações não necessariamente representam déficit hídrico, sendo necessários estudos mais detalhados a esse respeito.

Para os dois anos avaliados neste trabalho, a produção anual de sementes $\left(117 \mathrm{~kg} \cdot \mathrm{ha}^{-1}\right.$ e $160 \mathrm{~kg} \cdot \mathrm{ha}^{-1}$ para 2001 e 2002, respectivamente) esteve abaixo da estimada por Solórzano Filho (2001) na mesma região (427 kg.ha-1 em 1998) como também foi inferior à produção estimada em Santa Catarina (75 kg por planta) por Guerra et al. (2002). As diferenças, no primeiro caso, estão relacionadas principalmente à maior densidade de plantas reprodutivas femininas (46 indivíduos.ha ${ }^{-1}$ ) encontradas por Solórzano Filho (2001), quando comparadas a este estudo (18 indivíduos.ha ${ }^{-1}$ ), e também, a diferenças no método de estimativa de produção ou ainda à alternância entre anos de produção, como discutido anteriormente. Para o segundo caso, as diferenças também podem estar relacionadas à densidade de plantas: em Santa Catarina as plantas estavam em campo aberto, em baixa densidade, condições em que a produção de estróbilos por planta é maior do que nos remanescentes florestais (Guerra et al. 2002).

Neste estudo encontramos a relação entre elementos férteis e estéreis no estróbilo feminino de 1:6, semelhante à relação de 1:7 relatada por Hertel (1976) em araucária no Paraná. Este autor encontrou 6,4\% de pinhões chochos, bem acima do encontrado no presente estudo (1,6\%). Estes pinhões chochos, resultado da nãofertilização ou de aborto de óvulos, apresentam tamanho de uma semente sendo, no entanto, vazios, sem endosperma primário e embrião.

A reduzida predação de sementes pré-dispersão na área de estudo $(0,7 \%)$ indica que este fator afeta pouco a disponibilidade de sementes para a dinâmica da regeneração natural. Hertel (1976) encontrou 7,3\% das sementes atacadas pela mesma larva em araucária. Para Pinus resinosa, Mosseler et al. (1992) relatam variações de $11 \%$ a $93 \%$ na proporção de sementes, danificadas por uma larva de Lepidóptera, entre locais e anos. Segundo Crawley (2000), a predação prédispersão, em geral, apresenta grandes variações entre anos. A avaliação apenas da predação pré-dispersão, realizadas neste estudo, certamente está subestimando os valores de predação total. Solórzano Filho (2001) encontrou até $20,8 \%$ de predação pós-dispersão em araucária.

Este estudo demonstrou que o ciclo reprodutivo de A. angustifolia não é maior que dois anos na área de estudo nos anos avaliados. Devido à variação na amplitude de oferta e quantidade de sementes, a utilização racional deste recurso só é viável a partir de avaliações anuais do comportamento reprodutivo. Acompanhamento do comportamento reprodutivo em áreas de conservação e outros remanescentes permitirão estimar a quantidade, qualidade e período de oferta de sementes, informação necessária para tomada de decisões sobre o manejo e utilização adequada deste recurso.

Agradecimentos - Somos gratos ao Instituto Florestal do Estado de São Paulo, pela licença de pesquisa no Parque Estadual Campos do Jordão; à Fundação de Amparo a Pesquisa no Estado de São Paulo - Fapesp (95/9626-0), ao CNPq (582920/2001-9) e Capes pelo auxílio finaceiro e a dois revisores anônimos pelas sugestões. A. Mantovani recebeu bolsa de doutorado da Capes e L.P.C. Morellato e M.S. Reis são bolsistas de Produtividade em Pesquisa do CNPq.

\section{Referências bibliográficas}

ARISTA, M., ORTIZ, P.L. \& TALAVERA, S. 1997. Reproductive isolation of two sympatric subspecies of Juniperus phoenicea (Cupressaceae) in southern Spain. Plant Systematics and Evolution 208:225-237.

BACKES, A. 1999. Condicionamento Climático e distribuição geográfica de Araucaria angustifolia (Bertol.) Kuntze no Brasil - II. Pesquisa (Botânica) 49:31-51.

BISWAS, C. \& JOHRI, B.M. 1997. The Gymnosperms. Narosa Publishing House, New Dehli.

BIODIVERSITY SUPPORT PROGRAM. 1995. A regional analysis of geographic priorities for biodiversity conservation in Latin America and Caribbean. United States Agency for International Development, Washington.

BURLINGAME, L.L. 1914. The morphology of Araucaria brasiliensis. II. The ovulate cone and female gametophyte. Botanical Gazette 57:490-507.

CARVALHO, P.E.R. 1994. Espécies Florestais Brasileiras: recomendações silviculturais, potencialidades e uso da madeira. Empresa Brasileira de Pesquisa Agropecuária, Centro Nacional de Pesquisa de Florestas. EmbrapaCNPF, Colombo.

CRAWLEY, M.J. 2000. Seed predation and plant population dynamics. In The Ecology of Regeneration in Plant Communities (M. Fenner, ed.). Cabi Publishing, New York, p.167-182. 
DESPLAND, E. \& HOULE, G. 1997. Climate influences on growth and reproduction of Pinus banksiana (Pinaceae) at the limit of the species distribution in eastern North America. American Journal of Botany 84:928-937.

DINERSTEIN, E., OLSON, J.M., GRAHAM, D.J., WEBSTER, A. L., PRIIM, S.A., BOOKNDER, M.P. \& LEDEC, G. 1995. Una evaluación del estado de conservación de las ecoregiones terrestres de America Latina y el Caribe. Fondo Mundial para la Natureza, Banco Mundial, Washington.

EMBRAPA. 1998. Zoneamento ecológico para plantios florestais no Estado de Santa Catarina. CNPF-Embrapa, Curitiba.

ESPINDOLA, L.S., NOIN, M., CORBINEAU, F. \& CÔME, D. 1994. Cellular and metabolic damage induced by desiccation in recalcitrant Araucaria angustifolia embryos. Seed Science Research 4:193-201.

FERREIRA, A.G. 1977. Araucaria angustifolia (Bert.) O. Ktze. Germinação da semente e desenvolvimento da plântula. Tese de doutorado, Universidade de São Paulo, São Paulo.

FOURNIER, L.A. 1974. Um método cuantitativo para la medición de características fenológicas em árboles. Turrialba 24:422-423.

GALLO, D., NAKANO, O., SILVEIRA NETO, S., CARVALHO, R.P.L., BATISTA, G.C., FILHO, E. B., PARRA, J.R.P., ZUCCHI, R.A., ALVES, S.B. \& VENDRAMIN, J.D. 1988. Manual de Entomologia Agrícola. Editora Agronômica. Ceres Ltda, São Paulo.

GUERRA, M.P., SILVEIRA, V., REIS, M.S. \& SCHNEIDER, L. 2002. Exploração, manejo e conservação da araucária (Araucaria angustifolia). In Sustentável Mata Atlântica: a exploração de seus recursos florestais (L.L. Simões \& C.F. Lino, orgs.). Senac, São Paulo, p.85-102.

HAINES, R.J., PRAKASH, N. \& NIKLES, D.G. 1984. Pollination in Araucaria Juss. Australian Journal of Botany 32:583-594

HERTEL, R.J.G. 1976. Estudos sobre Araucaria angustifolia II. A constituição do estróbilo. Acta Biologica Paranaense 5:3-25.

HILTON-TAYLOR, C. 2000. IUCN red list of threatened species. IUCN, Gland.

HOULE, G. \& FILION, L. 1993. Interannual variations in the seed production of Pinus banksiana at the limit of the species distribution in Northern Quebec, Canada. American Journal of Botany 80:1242-1250.

INOUE, M.T., RODERJAN, C.V., KUNIOSHI, Y.S. 1984. Projeto Madeira do Paraná. Fupef, Curitiba.

JANZEN, D.H. 1967. Synchronization of sexual reproductive of trees within the dry season in Central America. Evolution 21:620-637.

JORDANO, P. 1991. Gender variation and expression of monoecy in Juniperus phoenicea (L.) (Cupressaceae). Botanical Gazette 152:476-485.
KELLY, D. 1994. The evolutionary ecology of mast seeding. Trends in Ecology and Evolution 9:465-470.

LIMA, A.R. \& CAPOBIANCO, J.P.R. (coords.). 1997. Mata Atlântica: avanços legais e institucionais para sua conservação. Documentos do ISA n. 004. Instituto Sócio Ambiental, Brasília.

LONGMAN, K.A. \& JENIK, J. 1974. Tropical forest and its environment. Longman Scientific \& Technical, New York.

MATTOS, J.R. 1994. O Pinheiro Brasileiro. 2 ${ }^{\mathrm{a}}$ ed. Artes Gráficas Princesa Ltda, Lages, v.1.

MOSSELER, A., ROBERTS, B.A. \& TRICCO, P. 1992. The effects of fir coneworm, Dioryctria abietivorella (Grote) (Lepidoptera: Pyralidae), on seed production in small, isolated populations of red pine, Pinus resinosa Ait. Forest Ecology and Management 53:15-27.

OFFORD, C.A., PORTER, C.L., MEAGHER, P.F. \& ERRINGTON, G. 1999. Sexual reproduction and early plant growth of the Wollemi Pine (Wollemia nobilis), a rare and threatened Australian conifer. Annals of Botany 84:1-9.

ORTIZ, P.L., ARISTA, M. \& TALAVERA, S. 2002. Sex ratio and reproductive effort in the dioecious Juniperus communis subsp. alpina (Suter) Celak. (Cupressaceae) along na altitudinal gradient. Annals of Botany 89:205-211.

OWENS, J.N., CATALANO, G.L., MORRIS, S.J. \& AITKENCHRISTIE, J. 1995. The reproductive biology of Kauri (Agathis australis). Pollination and prefertilization development. Internacional Journal of Plant Science 156:257-269.

POWELL, G.R. 1977. Initiation and development of subterminal buds in Abies balsamea. Canadian Journal of Forest Research 7:258-262.

PRIMAK, R.B. 1987. Relations among flowers, fruits, and seeds. Annual Review of Ecology and Systematics 18:409-430.

REITZ, R., KLEIN, R.M. \& REIS, A. 1978. Projeto Madeira de Santa Catarina. Sellowia 28-30:1-320.

SEIBERT, P., NEGREIROS, O.C., BUENO, R.A., EMMERICH, W., MOURA NETTO, B.V., MARCONDES, M.A.P., CESAR, S.F., GUILLAUMON, J.R., MONTAGNA, R.A.A., BARRETO, N.J.C.B., GARRIDO, M.A.O., MELLO FILHO, L.E., EMMERICH, M., MATTOS, J.R., OLIVEIRA, M.C. \& GODOI, A. 1975. Plano de manejo do Parque Estadual Campos de Jordão. Boletim Técnico do Instituto Florestal 19:1-148.

SHIMOYA, S. 1962. Contribuição ao estudo do ciclo biológico de Araucaria angustifolia (Bertoloni) O. Kuntze. Experientiae 2:520-540.

SIEGEL, S. 1975. Estatística não-paramétrica: para ciências do comportamento. Makron, São Paulo. 
SILVERTOWN, J.W. 1980. The evolutionary ecology of mast seeding in trees. Biological Journal of Linnean Society 14:235-250.

SOLÓRZANO FILHO, J. A. 2001. Demografia, fenologia e ecologia da dispersão de sementes de Araucaria angustifolia (Bert.) O. Kuntze (Araucariaceae), numa população relictual em Campos do Jordão. Dissertação de mestrado, Universidade de São Paulo, São Paulo.
STEEL, R.G.D., TORRIE, J.H. \& DICKEY, D.A. 1997. Principes and procedures of statistics a biometrical approach. $3^{\text {rd }}$ ed., McGraw-Hill, New York.

VAN SCHAIK, C.P., TERBORGH, J.W. \& WRIGHT, S.J. 1993. The phenology of tropical forest: adaptative significance and consequences of consumers. Annual Review of Ecology and Systematics 24:353-377.

ZAR, J.H. 1996. Biostatical analysis. Prentice-Hall, New Jersey. 